\title{
LHCf physics results and future prospectives
}

\author{
Alessia TRICOMI* on behalf of the LHCf Collaboration \\ University of Catania and INFN Catania, Italy \\ E-mail: alessia.tricomiect.infn.it
}

The LHCf experiment has been designed to precisely measure forward neutral particle spectra produced in proton-proton collisions at LHC up to an energy of $14 \mathrm{TeV}$ in the center of mass system. These measurements are of fundamental importance to calibrate the Monte Carlo models widely used in the high energy cosmic ray (HECR) field, up to an equivalent laboratory energy of the order of $10^{17} \mathrm{eV}$.

In 2009-2010 the experiment has completed the p-p data taking at $\sqrt{s}=0.9 \mathrm{TeV}$ and $\sqrt{s}=7 \mathrm{TeV}$ and the detectors have later on been removed from the tunnel region, when the LHC luminosity increased above $10^{30} \mathrm{~cm}^{-2} \mathrm{~s}^{-1}$. The detectors are now being upgraded in view of the p-p run at $\sqrt{s}=14 \mathrm{TeV}$.

In this paper the first results on the inclusive photon spectrum measured by LHCf is reported. Comparison of this spectrum with the model expectations show significant discrepancies, mainly in the high energy region.

In addition, perspectives for future analyses as well as the program for the next data taking period will be discussed.

The 2011 Europhysics Conference on High Energy Physics-HEP 2011,

July 21-27, 2011

Grenoble, Rhône-Alpes France

${ }^{*}$ Speaker. 


\section{The LHCf Experiment}

The LHCf experiment is composed by two independent position sensitive electromagnetic calorimeters, located on both side of the ATLAS experiment, $140 \mathrm{~m}$ away from the LHC-IP1 interaction point. Each calorimeter (ARM1 and ARM2) has a two tower structure, with the smaller tower located at zero degree collision angle, approximately covering the region with pseudorapidity $\eta>10$ and the larger one, approximately covering the region with $8.4<\eta<10$. Four X-Y layers of position sensitive detectors (scintillating fibers in ARM1, silicon micro-strip detectors in ARM2) provide measurements of the transverse profile of the showers. The two tower structure allows to reconstruct the $\pi^{0}$ decaying in two $\gamma \mathrm{s}$, hitting separately the two towers, hence providing a very precise absolute energy calibration of the detectors. In the range $\mathrm{E}>100 \mathrm{GeV}$, the LHCf detectors have energy and position resolutions for electromagnetic showers better than $5 \%$ and $200 \mu \mathrm{m}$, respectively. A detailed description of the LHCf experimental set-up and of the expected physics performances can be found in Ref. [1]. The experiment has recorded on disk more than $10^{5}$ events in the $\sqrt{s}=900 \mathrm{GeV}$ runs and more than $3 \times 10^{8}$ events in the $\sqrt{s}=7 \mathrm{TeV}$ runs, allowing a precise measurement of the photon and $\pi^{0}$ spectra forward produced up to the maximum accelerator energies. The detectors have been removed from the LHC tunnel during summer 2010, when the luminosity raised above $10^{30} \mathrm{~cm}^{-2} \mathrm{~s}^{-1}$, to avoid significant radiation damage.

\section{The single photon energy spectrum}

A measurement of the single photon energy spectrum at $7 \mathrm{TeV}$ p-p collisions has been recently published by the LHCf Collaboration [2]. Here we briefly summarize the main steps of the analysis with special emphasis to possible implication for the calibration of Monte Carlo models used in HECR Physics. Only a small subset of the $7 \mathrm{TeV}$ p-p collision data has been used for this first analysis, corresponding to an integrated luminosity of $0.68 \mathrm{nb}^{-1}$ and $0.52 \mathrm{nb}^{-1}$ for the ARM1 and ARM2 detectors, respectively. The analysed data have been chosen in a particularly clean and low luminosity fill, to minimize backgrounds, hence reducing the systematics of the measurement.

The energy of photons is reconstructed from the signal released by the shower particles in the scintillators, after applying corrections for the non-uniformity of light collection and for particles leaking in and out of the edges of the calorimeter towers. In order to correct for these last two effects, which are rather important due to the limited transverse size of both the calorimetric towers, the transverse impact position of showers provided by the position sensitive detectors is used.

Event produced by neutral hadrons are rejected applying a simple algorithm based on the longitudinal development of the showers, which is different for electromagnetic and hadronic particles. In addition, thanks to the information provided by the position sensitive detectors, events with more than one shower inside the same tower (multi-hit) are rejected. In order to combine the spectra measured by ARM1 and ARM2, which have different geometrical configurations, in this analysis only events detected in a common pseudo-rapidity and azimuthal range are selected: $\eta>10.94$ and $\Delta \phi=360^{\circ}$ for the small towers and $8.81<\eta<8.99$ and $\Delta \phi=20^{\circ}$ for the large towers. Figure 2 shows the single $\gamma$ spectrum measured by LHCf in the two pseudo-rapidity regions compared with results predicted by MC simulations using different models: DPMJET 3.04 [3], QGSJET II-03 [4], SIBYLL 2.1 [5], EPOS 1.9 [6] and PYTHIA 8.145 [7]. Statical errors and systematic uncertainties 

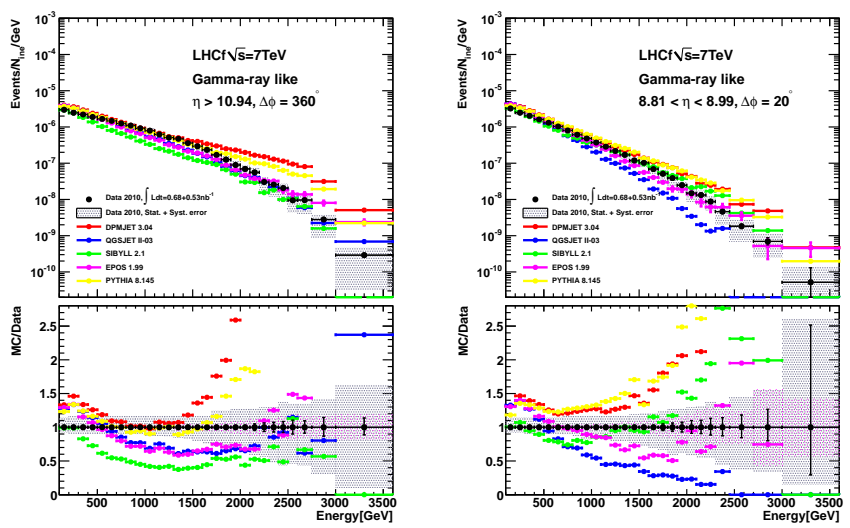

Figure 1: Single photon energy spectra measured by LHCf (black dots) for $\eta>10.94$ (left) and $8.81<$ $\eta<8.99$ (right) compared to the predictions of DPMJET 3.04 (red), QGSJET II-03 (blue), SIBYLL 2.1 (green), EPOS 1.99 (magenta) and PYTHIA 8.145 (yellow). Top panels show the spectra and bottom panels show the ratio of MC results to experimental data. Error bars and gray shaded areas in each plot indicate the statistical and the systematic errors, respectively. The blue shaded area indicates the statistical error of the MC data. Figure from Ref. [2].

are also plotted. A careful study of systematic uncertainties has been done and conservative estimates have been taken into account. Further details can be found in Ref. [2]. As can be seen from Fig. 2, a clear discrepancy between the experimental results and the predictions of the models in the whole energy region is present.

\section{Future perspectives}

The first LHCf results on the photon energy spectra have raised attention in the HECR community. A collaboration with several MC developers and theoreticians has started to better understand the implication of the LHCf results in HECR Physics and to address the importance of future measurements. As an example, we have artificially modified the QGSJET II model to produce a $\pi^{0}$ spectrum that differs from the original one by an amount approximately equal to the difference expected between the different models. Figure 2 shows the average longitudinal development of the atmospheric shower in the original QGSJET II model (red points) and in the artificially modified model (blue points). A difference in the position of the shower maximum of the order of $30 \mathrm{~g} / \mathrm{cm}^{2}$ is observed. As can be seen from the plot, the large Feynman $x_{F}\left(x_{F}>0.1\right)$ particles, which are the ones experimentally accessible by LHCf, are the dominant component of the air shower development. A shift of the order of $30 \mathrm{~g} / \mathrm{cm}^{2}$ in the average position of the shower maximum $\left.\left(<X_{\max }\right\rangle\right)$, which is the most used method to infer cosmic rays composition, may reflect significantly in the interpretation of HECR data. Fig. 3 shows, as an example, the Auger results [8] for the $\left\langle X_{\max }\right\rangle$ variable as function of the energy compared with the model predictions for a proton-like (red lines) and an Iron-like (blue lines) cosmic ray components, respectively. The yellow rectangle correspond to the $30 \mathrm{~g} / \mathrm{cm}^{2}$ shift, obtained in Fig. 2. The importance of a direct measurement of the $\gamma$ and $\pi^{0}$ spectra by LHCf is clear. 


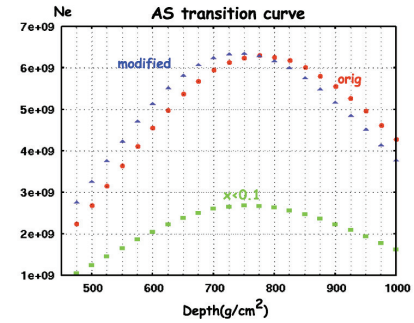

Longitudinal AS development

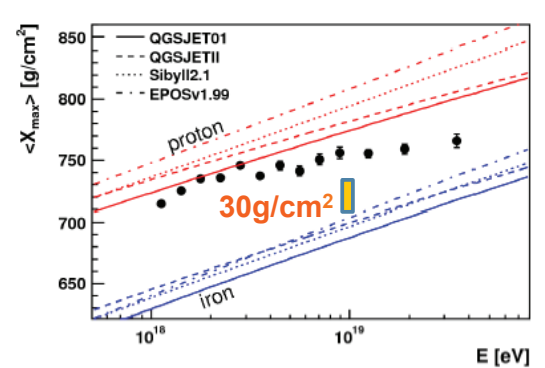

Figure 2: Average longitudinal development of the atmospheric shower in the QGSJET II model (red points) and in the artificially modified model (blue points).
Figure 3: $\left\langle X_{\max }\right\rangle$ distribution as measured by Auger [8] (black points) compared with the model expectations for a light (red) or heavy (blue) cosmic ray composition. The yellow rectangle correspond to the $30 \mathrm{~g} / \mathrm{cm}^{2}$ shift, obtained in Fig. 2.

New analyses of data collected in 2009-2010 runs are in progress, in particular the measurement of the single photon spectra at $900 \mathrm{GeV}$ as well as the reconstruction of $\pi^{0}$ spectra.

In the meantime, the LHCf Collaboration is also working on the upgrade of the detector to improve the radiation resistance in view of the $14 \mathrm{TeV}$ p-p run, currently foreseen in 2014. The scintillating part of the detector will be replaced with GSO slabs. Beam test results [9] have demonstrated that the upgrade of the calorimeter with GSO will enable LHCf to sustain the radiation level foreseen in the $14 \mathrm{TeV}$ run. Additional improvements in the front-end electronics of the silicon position sensitive layers of ARM2 detectors as well as an optimization of the layout to improve the stand-alone silicon energy resolution are on going.

We are also investing the Physics implication of taking data in $\mathrm{p}-\mathrm{Pb}$ collisions which could become feasible at the end of 2012.

\section{References}

[1] O. Adriani, et al., The LHCf detector at the CERN Large Hadron Collider, JINST 3 (2008) S08006.

[2] O. Adriani, et al., Measurement of zero degree single photon energy spectra for $\operatorname{sqrt}(s)=7 \mathrm{TeV}$ proton-proton collisions at LHC, Phys. Lett. B703 (2011) 128.

[3] F.W. Bopp, et al., Antiparticle to Particle Production Ratios in Hadron-Hadron and d-Au Collisions in the DPMJET-III Monte Carlo, Phys. Rev. C77 (2008) 014904.

[4] S. Ostapchenko, Monte Carlo treatment of hadronic interactions in enhanced Pomeron scheme: I. QGSJET-II model., Phys. Rev. D83 (2011) 014108.

[5] E.-J. Ahn, et al., Cosmic ray interaction event generator SIBYLL 2.1, Phys. Rev. D80 (2009) 094003.

[6] K. Werner, et al., The hadronic interaction model EPOS, Nucl.Phys.Proc.Suppl. 175-176 (2008) 81.

[7] T. Sjöstand, et al., A Brief Introduction to PYTHIA 8.1, Comput. Phys. Comm. 178 (2008) 852.

[8] J.Bellido for the Auger Collaboration, The distribution of shower maxima of UHECR air-showers in proceedings of $32^{\text {st }}$ ICRC, Beijing, (2011).

[9] K. Kawade, et al., Study of Radiation Hardness of $\mathrm{Gd}_{2} \mathrm{SiO}_{5}$ scintillator for Heavy Ion Beam, JINST 6 (2011) T09004. 